Volume 10, No.3, May - June 2021

International Journal of Advanced Trends in Computer Science and Engineering

Available Online at http://www.warse.org/IJATCSE/static/pdf/file/ijatcse141032021.pdf

https://doi.org/10.30534/ijatese/2021/141032021

\title{
On k-Distance Degree Index of Trees
}

\author{
Zia Ullah Khan ${ }^{1}$, Azhar Iqbal ${ }^{2}$, Gohar $\mathrm{Ali}^{3}$, Marium Sultana ${ }^{4}$, Javed Khan ${ }^{5}$ \\ ${ }^{1}$ School of Mathematical Sciences. Shanahai Jiao Tong University. 800 Dongchuan Road, Shanghai, 2000240, PR China. \\ z.u.khan@sjtu.edu.cn \\ ${ }^{2}$ Department of Basic Sciences. Dawood University of Engineering and Technology, MA Jinnah road, Karachi, Pakistan. \\ azhar.iqbal@duet.edu.pk \\ ${ }^{3}$ Department of Mathematics, Islamia College Peshawar, Peshawar, Khyber Pakthunkhwa, Pakistan. \\ gohar.ali@icp.edu.pk \\ ${ }^{4,5}$ Department of Mathematical Sciences. Federal Urdu University of Arts, Science and Technology, Gulshan Iqbal, Karachi, \\ Pakistan. \\ marium.sultana@fuuast.edu.pk,javid.khan@fuuast.edu.pk \\ Corresponding Author: azhar.iqbal@duet.edu.pk
}

\begin{abstract}
Let $\mathrm{G}$ be a connected graph then $N_{k}$-index ( $k$-distance degree index) defined in [14] as $N_{k}(G)=\sum_{k=1}^{\operatorname{diam}(G)}\left(\sum_{x \in V(G)} d_{k}(x)\right) k$, where $d_{k}(x)=$ $\left|N_{k}(x)\right|=|\{y \in V(G): d(x, y)=k\}|$, where $d(x, y)$ is the distance between vertex $x$ and $y$ in graph $G$ and $\operatorname{diam}(G)$ is the diameter of graph $G$. We define some transformations and their impact on $N_{k}$-index of graphs with respect to pendant path and pendant vertices. For fixed number of pendant vertices of a tree, we define a tree with minimum $N_{k}$-index. Also for different fixed parameters we characterize the tress with minimum $N_{k}$-index.
\end{abstract}

Keywords: Broom Graph, Distances in graphs, Distance topological index, $N_{k}$-index, Trees.

\section{INTRODUCTION}

Let $G$ be a simple connected graph of order $|V|=n$ and sizel $E \mid=m$. Let $x, y \in V(G)$ be any two vertices of $G$, then $d(x, y)$ is length of the shortest path between them, known as distance from $x$ to $y$ and vice versa. The number of adjacent vertices or neighbors to a vertex $x \in G$ is called degree of $x$ and denoted as $\operatorname{deg}(x)$. A vertex having only one neighbor or degree 1 is known as pendent vertex or leaf. If $x \in V(G)$ such that its degree is maximum among all vertices of the graph, then its degree is denoted by $\Delta$ or $\Delta(G) . \Delta(G)$ is called maximum degreeof $G$. Let $D(G)=$ $\left[d_{G}(x y)\right]$ be the distance matrix of $G$, where $d_{G}(x y)=$ $d(x, y)$. The maximum distance between any two vertices in a graph is called diameter and denoted as $\operatorname{diam}(G)$.

Let $\mathrm{k}$ be a positive integer and $x \in V(G)$, we define the open $\mathrm{k}$ neighborhood of $\mathrm{x}$ in $\mathrm{a}$

For a vertex $x \in V(G)$ and a positive integer $k$ the open $k$ neighborhood of $x$ in a graph $G$ is denoted by $N_{k}(x)$ and is defined as $N_{k}(x)=\{y \in V(G): d(x, y)=k\}$ [16], [17]. A matching $M$ is a set of pairwise nonadjacent edges in $G$. If set $M$ matches all the vertices of $G$ then it is known as perfect matching.

The distance and adjacency based topological indices are mathematically extracted from the structure of graph. In most cases it is derived from the chemical graphs where the graphs represents molecules or atoms, which usually does not depends on its pictorial representation of the graph and it is the graph invariant number calculated from a graph representing a molecule.

The topological indices are basically derived to establish and represent a relationship between the chemical properties, biological activity or most importantly the molecules. The topological indices are known to be distance-based topological indices if they are defined as the distance function in the molecule of compound structure study. The main role played in extracting distance based topological indices is of Distance matrix of a graph, see [2] along with a survey paper [3].

In literature there are numerous distance based topological indices studies in detail based on its applications in chemistry and computer science. The most detailed studied distance based topological index is Wiener Index [4], defined as:

$$
W(G)=\sum_{x, y \in V} \quad d(x, y)
$$

In 1992 another distance based topological index was introduced by Mihulic et al. [6] known as Harrary index.

$$
H(G)=\sum_{x, y \in V} \quad \frac{1}{(d(x, y))^{2}}
$$

but with passage of time the Harrary index nowadays is defined as [7],[8],

$$
H(G)=\sum_{x, y \in V} \quad \frac{1}{d(x, y)}
$$

Further in 1993 Randie'[5] introduced the hyper Wiener index, defined as;

$$
W W(G)=\sum_{(x, y) \epsilon V(G)} \quad\left(d^{2}(x, y)+d(x, y)\right)
$$

In 1989 a new distance based index named as Schultz Index defined by H. P. Schultz [9], which later on was known as degree distance index by A. Dobrynin et al. [10] and is denoted as $\mathrm{DD}(\mathrm{G})$. 


$$
S(G)=D D(G)=\sum_{(x, y) \epsilon V(G)} \quad(d(x)+d(y)) d(x, y)
$$

For detailed study the readers may refer to [3],[11],[12], [13] and the references therein.

Recently Naji et al. [14], [15],[18],[19] in their paper defined a new distance based index called $N_{k}$-index of graph or k-distance degree index of graph. They proposed some general $N_{k}$-index of common graphs and provided some bounds on $N_{k}$-index of graphs. In [15] they computed and proved some results of $N_{k}$-index of certain graph operations.

Our goal here is to further support and add some results to $N_{k}$-index of graphs. In graph theory, it is important to study trees with respect to different parameters and generally bounded trees with maximum degree. In section 1 of this paper we just introduced some basic knowledge of distance based topological indices and their applications. In section 2 we will discuss some known lemmas. In section 3 we define and prove some transformations and their impact on $N_{k}$-index of graphs with respect to pendant path and pendant vertices. The lemmas of section 2 will be often used in following section as an argument to main results. In section 4 we characterize the tree with minimum $N_{k}$-index for fixed pendant vertices and determine the minimum $N_{k^{-}}$ index of trees in terms of their domination number, independence number and matching number. Finally in section 5 we define a tree $A_{n, \Delta}$ (a tree made from two disjoint stars of same degree $S_{\Delta}$ by adding an edge between their centers and to any pendant vertex of star attaching pendant path of $\mathrm{n}-2 \Delta$ vertices, where $n \geq 2 \Delta+1 \geq 7$ and prove that $A_{n, \Delta}$ attains the maximum $N_{k}$-index.

\subsection{Definition}

For a connected graph $G$ on $\mathrm{n}$ the $N_{k}$-index of $G$ is defined as

$$
N_{k}(G)=\sum_{k=1}^{\operatorname{diam}(G)} \quad\left(\sum_{x \in V} \quad d_{k}(x)\right) k,
$$

\subsection{Example}

Let $G$ be a graph with 5 vertices as shown in Figure 1 .

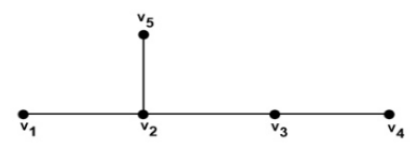

Figure 1: Connected Graph

$\operatorname{diam}(G)=3$.

$$
\begin{aligned}
& N_{k}(G)=\sum_{k=1}^{\operatorname{diam}(G)}\left(\sum_{x \in V} d_{k}(x)\right) \cdot k \\
&=\left(\sum_{x \in V} d_{1}(x)\right) \cdot 1+\left(\sum_{x \in V} d_{2}(x)\right) \cdot 2 \\
&+\left(\sum_{x \in V} d_{3}(x)\right) \cdot 3 \\
& N_{k}(G)=(1+3+2+1+1)(1)+(2+1+2+1 \\
&+2)(2)+(1+0+0+1+1)(3)=33
\end{aligned}
$$

\section{PRELIMINARIES}

In this section we focus on some already known results which will support the in proving the argument for our main result.

Lemma 2.1 [1] For any bipartite graph $G$ the matching number equals the minimum cardinality of covering of $G$.

Lemma 2.2 [14] For a connected graph $G$ with $n$ vertices and $m$ edges and $\operatorname{diam}(G)=2$,

$$
N_{k}(G)=2 n(n-1)-2 m .
$$

Lemma 2.3[14] Let $G$ be $n$ order connected graph with $\operatorname{diam}(G)=2$. Then

$$
(\operatorname{diam}(G)+1) \operatorname{diam}(G) \leq N_{k}(G) \leq n(n-1) \operatorname{diam}(G)
$$

Lemma 2.4 [14] Let $G$ be $n$ order connected graph. Let $e$ be an edge such that $e$ is not a cut edge of $G$. Then

$$
N_{k}(G) \leq N_{k}(G-e)
$$

Lemma 2.5 [14] Let $G$ be n order connected graph such that $G ¥ K_{n}$. Then

$$
N_{k}\left(K_{n}\right)<N_{k}(H)
$$

Lemma 2.6 Let $G$ be $\mathrm{n}$ order connected graph. Let $\mathrm{H}$ be a connected spanning subgraph of $G$. Then

$$
N_{k}(G)<N_{k}(H)
$$

Naji et al. [14] calculated generalized $N_{k}$-indexof complete graph $K_{n}$ cycle graph $C_{n}$ and a path graph $P_{n}$ for $n \geq 2$ as:

$$
\begin{gathered}
N_{k}\left(K_{n}\right)=n(n-1), N_{k}\left(P_{n}\right)=\frac{n^{3}-n}{3} \text { and } \\
N_{k}\left(C_{n}\right)=\left\{\frac{n^{3}}{4} \text { ifn iseven } \frac{n\left(n^{2}-1\right)}{4} \text { if } n\right. \text { isodd }
\end{gathered}
$$

\section{SOME GRAPH TRANSFORMATION}

Let $G$ be $n$ order connected graph. Let $x y=e \in E(G)$, then $G-x y$ is the graph by deleting an edgexy, while $G+x y$ is the graph transformed from $G$ by connecting vertex $x$ and vertex $y$ with an edge. A path with all internal vertices of degree two and one end vertex of degree one is known as pendant path.

In this section we propose transformations on $N_{k}$ indexof trees in terms of pendant paths and pendant vertex.

Theorem 3.1 Let $t \in V(G)$ be a vertex of nontrivial graph $G$ and $p \geq q \geq 1$, where $p, q \in Z$. A graph $G(p, q)$ obtained from $G$ by connecting two pendant paths $P$ and $Q$ of length $p$ and $q$ (see figure 2) that is

$$
P:=t u_{1} u_{2} \ldots u_{p} \text { and } Q:=t v_{1} v_{2} \ldots v_{p} \text { respectively. }
$$

Then the following inequality holds:

$$
N_{k}(G(p, q))<N_{k}(G(p+1, q-1))
$$


Proof: As when we move from $G(p, q)$ to $G(p+1, q-1)$ the the number of vertices and edges remains the same.

Let us denote by $P^{\prime}$ and $Q^{\prime}$ the paths $t u_{1} u_{2} \ldots u_{p} v_{q}$ and $t v_{1} v_{2} \ldots v_{p-1}$ respectively. We see that the paths $P \cup Q$ and $P^{\prime} \cup Q^{\prime}$ are both isomorphic to each other and are of length $\mathrm{p}+\mathrm{q}+1$. Now for any $v \in P \cup Q$ and $v^{\prime} \in P^{\prime} \cup Q^{\prime}$ by shifting $P \cup Q$ for one place as they are isomorphic to $P_{p+q+1}$. Now for any $v \in P \cup Q$ and $v^{\prime} \in P^{\prime} \cup Q^{\prime}$ by shifting $P \cup Q$ for one place as both unions are isomorphic to the path of length $p+q+1$. From above argument we have $N_{k}(P \cup$ $Q)=N_{k}\left(P^{\prime} \cup Q^{\prime}\right)$.

Now for any vertex $u \in G$ we have the following result:

$$
\begin{gathered}
d_{v \in(P \cup Q) \backslash v_{p}}(u, v)=d_{v \in\left(P^{\prime} \cup Q^{\prime}\right) \backslash v_{p}}(u, v) \\
\Rightarrow N_{k}\left(G(p, q) \backslash\left\{v_{p}\right\}\right)= \\
N_{k}\left(G(p+1, q-1) \backslash\left\{v_{p}\right\}\right)
\end{gathered}
$$

As $p \geq q \geq 1$, then for any vertex $v \in G, d\left(v, v_{p}\right)$ in $G(p, q)$ will always be strictly less that $d\left(v, v_{q}\right)$ in $G(p+$ $1, q-1)$. From which

Follows

$$
\begin{gathered}
\sum_{v \in V(G(p, q) \backslash P \cup Q)} d_{k}(v)< \\
\sum_{v \in V(G(p+1, q-1)) \backslash P^{\prime} \cup Q^{\prime}} d_{k}(v)
\end{gathered}
$$

By combining all the above arguments we see that

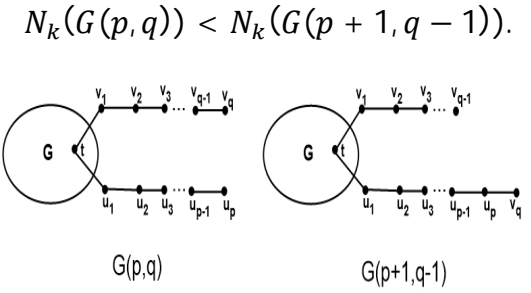

Figure 2: Non Trivial Graphs

The result in lemma 3.1 remains true if the vertex $t$ is replaced by an edge.

\subsection{Corollary}

Let $\mathrm{x}, \mathrm{y} \in \mathrm{V}(\mathrm{G})$ such that $\mathrm{x} \sim \mathrm{y}$. Let $\mathrm{p} \geq \mathrm{q} \geq 1$ and $\mathrm{G}(\mathrm{p}, \mathrm{q})$ be the graph transformed from $\mathrm{G}$ by connecting distinct pendant paths $\mathrm{P}$ and $\mathrm{Q}$ of length $\mathrm{p}$ and $\mathrm{q}$ respectively (see figure 2) i.e $P=x u_{1} u_{2} \ldots u_{p}$ and $Q=y v_{1} v_{2} \ldots v_{p}$ at vertex $\mathrm{x}$ and $\mathrm{y}$, respectively. Then

$$
N_{K}(G(p, q))<N_{K}(G(p+1, q-1))
$$

\subsection{Lemma}

Let $\mathrm{G}$ and $G^{*}$ be connected bipartite graphs (see figure $3)$, where
$P=v \ldots w$ denotes a unique pendant path starting at vertex v. We can obtain graph $G^{*}$ from $G$ by switching $N(v)$ in $\mathrm{V}(\mathrm{Q})$ and connected them to vertex $\mathrm{u}$. If

$$
d_{G}(v, w) \leq \max \{d(u, y) ; y \in V(S) \text { and } \quad \mathrm{S} \text { is not a }
$$
pendant path starting at vertex $\mathrm{u}$, then

$$
N_{K}(G)=N_{K}\left(G^{*}\right)
$$

\section{Proof:}

Let $G$ and $G^{*}$ be connected bipartite graphs (see figure 3). Let $G^{*}$ denotes the graph obtained form $\mathrm{G}$ by switching $\mathrm{N}(\mathrm{v})$ in $\mathrm{V}(\mathrm{Q})$ to vertex $\mathrm{u}$. let $P=v \ldots w$ be the only pendant path. As we move from $G$ to $G^{*}$, the number of vertices is unchanged.

Since

$$
d_{G}(v, w) \leq \max \{d(u, y) ; y \in V(S)\}
$$

let $\mathrm{x} \in \mathrm{V}(\mathrm{S})$, such that $d_{G}(v, w) \leq d_{G}(v, w) \quad$ i.e $d_{P}(v, w) \leq d_{S}(v, w)$. As $\mathrm{S}$ itself is not just a pendant path, whose end vertex is $\mathrm{u}$, its obvious that $\mathrm{P}$ is the subgraph of $S$. From graphs in figure 3 , for any $t \in V(P)$,

$$
\begin{gathered}
\sum_{t \in V(P)} \quad d_{k}^{G}(t)<\sum_{t \in V(P)} \quad d_{k}^{G^{*}}(t), \text { where } \\
k=1,2, \ldots, d(G)
\end{gathered}
$$

Similarly we see that for any vertex $t \in \mathrm{V}(\mathrm{S})$, $\sum_{t \in V(S)} \quad d_{k}^{G}>\sum_{t \in V(S)} \quad d_{k}^{G^{*}}$. As $\mathrm{P}$ is induced in $\mathrm{S}$, so let $Q=u, \ldots, y$ be the path of same length in $\mathrm{V}(\mathrm{S})$ as of P. Now we see for $t \epsilon \mathrm{P}$ the distance with $\mathrm{V}(\mathrm{Q})$ is increased by 1 but for each $t \in P$ there exist a vertex $x \in Q$ whose distance is decreased with $\mathrm{V}(\mathrm{Q})$ by 1 . As $\mathrm{V}(\mathrm{S})$ also is non pendant path so the distance with vertices not on $\mathrm{Q}$ is also decreased by 1 . The above arguments lead us to conclusion that for any $\mathrm{z} \epsilon$ $\mathrm{G}$ and $\mathrm{z} \in G^{*}, \sum_{z \in V(G)} \quad d_{k}^{G}(z)>\sum_{t \in V\left(G^{*}\right)} \quad d_{k}^{G^{*}}(z)$. So we conclude $N_{k}(G)>N_{k}\left(G^{*}\right)$.

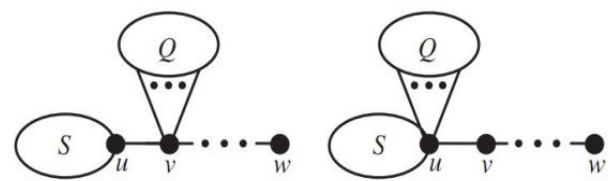

Figure 3: Connected Bipartite Graphs

\section{$4 \quad N_{k}$-INDEX OF TREES WITH FIXED} PARAMETERS

Let $\mathrm{B}(\mathrm{n}, \mathrm{r})$ denotes all $\mathrm{n}$ vertex trees with $\mathrm{r}$ fixed pendant vertices and $2 \leq r \leq n-1$.

Let $n, r>0$. Let $s=\left\lfloor\frac{n-1}{r}\right\rfloor$ and $l=n-1-r s$. Let $T_{n, r}$ represents the trees where $r-1$ paths are attached to $s$ vertices and to $s+1$ vertices 1 number of paths are attached, where

$$
2 \leq r \leq n-1 \text {. }
$$

\subsection{Theorem}

Let $\mathrm{G} \in \mathrm{B}(\mathrm{n}, \mathrm{r})$, where $2 \leq r \leq n-2$. Then 


$$
N_{k}(G)>N_{k}\left(T_{n, r}\right) \text {. }
$$

where equality holds if $G \cong T_{n, r}$.

\section{Proof.}

Let $r$ denotes the number of pendant vertices in $G$, then for $r=2, n-1$ the cases are obvious and trivial i.e. path graph on $\mathrm{n}$ vertices and star graph on $\mathrm{n}$ vertices respectively. So we consider for $3 \leq r \leq n-2$.

Let $\mathrm{G} \in \mathrm{B}(\mathrm{n}, \mathrm{r})$ be a tree and assume that $\mathrm{G}$ has minimal $N_{k}$-index. Assume that $V^{*}(G)$ contains the vertices of $\mathrm{G}$ with minimum degree 3 . Let $\mathrm{x} \in V^{*}(G)$ and let pendant path $\mathrm{P}$ rooted at vertex $\mathrm{x}$ has a minimum length, and $\mathrm{y}$ be end vertex of $\mathrm{P}$ in $\mathrm{G}$.

Suppose $\left|V^{*}(G) \geq 2\right|$. By choice of vertex $\mathrm{z} \in V^{*}(G)$, such that $\mathrm{d}(\mathrm{x}, \mathrm{z})$ is possibly as small as can. Then vertices of the unique path (if exists) connecting $\mathrm{x}$ and $\mathrm{z}$ as is pendant in $\mathrm{G}$ are all of degree 2. Let $\mathrm{w} \in \mathrm{G}$ be adjacent to $\mathrm{x}$ on the path from $\mathrm{x}$ and $\mathrm{z}(\mathrm{x}=\mathrm{z}$ if $\mathrm{z}$ and $\mathrm{x}$ are adjacent). Let $\mathrm{S}$ represents the component of G-wx containing $\mathrm{w}$. As $\mathrm{S}$ is not a pendant path with end vertex $\mathrm{w}$, by path $\mathrm{P}$ we have $d(x, y) \leq \max \{d(w, z) ; z \in V(S)$. By lemma 3.3 to $\mathrm{G}$, we have $G^{*} \in B(n, r)$, such that

$$
N_{K}(G)>N_{K}\left(G^{*}\right),
$$

Which is also a contradiction. So $\mathrm{x}$ is the only vertex in $\mathrm{G}$ with degree at least 3 .

If $G ¥ T_{n, r}$, then by theorem 3.1 we see that $\quad N_{K}(G)>$ $N_{K}\left(T_{n, r}\right)$, which leads to contradiction. It follows that $G \cong T_{n, r}$,

\subsection{Theorem}

Let $2 \leq r \leq n-2$ be number of pendant vertices. Then $N_{K}\left(T_{n, r}\right)>N_{K}\left(T_{n, r+1}\right)$,

\section{Proof.}

Let $x \in T_{n, r}$, such that $\mathrm{x}$ is the end vertex of the longest pendant path in $T_{n, r}$. Let $\mathrm{y} \sim \mathrm{x}$ and $\mathrm{y} \sim \mathrm{z}$ (neighbor of $\mathrm{y}$ in $T_{n, r}$ different from $\left.\mathrm{x}\right)$. Then by theorem 3.1

$N_{K}\left(T_{n, r}\right)>N_{K}\left(T_{n, r}-x y+y z\right)$, as there are exactly $\mathrm{r}+1$ pendant vertices in $T_{n, r}-x y+x z$. By theorem 4.1,

$$
N_{K}\left(T_{n, r}-x y+x z\right)>N_{K}\left(T_{n, r+1}\right) .
$$

The result follows.

The set of vertices in $\mathrm{G}$ form a matching set $\mathrm{M}$, if no two edges in the vertex set of $\mathrm{M}$ has a common vertex or simply $\mathrm{M}$ contains pairwise non-adjacent edges. If set $\mathrm{M}$ matches all the vertices of $\mathrm{G}$ then is known as perfect matching.

Let $l$ be a positive integer such that $2 \leq l \leq\left\lfloor\frac{n}{2}\right\rfloor$. Let $A^{n, l}$ denotes the tree formed by connecting $l-1$ distinct paths on any 2 vertices to the center of the star $S_{n-2 l+2}$

\subsection{Lemma}

Let $\mathrm{G}$ be $\mathrm{n}$ order tree with matching number $\mathrm{m}$, where $2 \leq m \leq\left\lfloor\frac{n}{2}\right\rfloor$. Then $N_{k}(G) \geq N_{k}\left(A^{n, m}\right)$ where equality holds if $G \cong A^{n, m}$

Proof.

Let $\mathrm{G}$ be a graph on $\mathrm{n}$ vertices with matching number $\mathrm{m}$ and pendant vertices $r$. It is obvious that in $\mathrm{M}$ for one edge there must exist atleast pendant vertex. Then $r \leq m+(n-$ $2 m$ ). Now if $\mathrm{r}=\mathrm{n}-\mathrm{m}$, by theorem 4.1 (with $\mathrm{s}=1$ and $l=m-1$ ) we have $A^{n, m} \cong T_{n, n-m}$, a unique tree which has minimal $N_{k}$-index. Otherwise if $r<n-m$, by theorem 4.1 and theorem 4.2 the result follows as,

$$
N_{K}(G)>N_{K}\left(A^{n, m}\right)>N_{K}\left(A^{n, m-1}\right)>\cdots>N_{K}\left(A^{n, c}\right)
$$

A collection of vertices I such that $\mathrm{x}, \mathrm{y} \in \mathrm{I}$ and $\mathrm{x} ¥ \mathrm{y}$ is known to be independent set of graph G. The maximum size of independent set of $\mathrm{G}$ is known as its independence number and denoted by $a=a(G)$. [1] We have, for any bipartite graph $\mathrm{G}$, the independence number equals the matching number i.e vertex set of G. So from lemma 4.3 we have,

\subsection{Corollary}

Let $\mathrm{G}$ be $\mathrm{n}$ vertex tree with independence number $\mathrm{b}=$ $\mathrm{b}(\mathrm{G}), \quad$ where $\quad\left\lceil\frac{n}{2}\right\rceil \leq b \leq n-2 . \quad$ Then $\quad N_{K}(G) \geq$ $N_{K}\left(A^{n, n-b}\right)$, with equality if $G \cong A^{n, n-b}$.

Let $\mathrm{G}$ be $\mathrm{n}$ vertex graph then the subset of vertices in $\mathrm{G}$, whose neighborhood contains all distinct vertices of $G$ is called dominating set. We denote the domination number by $c=c(G)$ i.e., the minimum number of elements of a dominating set of $\mathrm{G}$. A vertex set $\mathrm{K}$ of $\mathrm{G}$ is known as covering of $G$ such that for each edge of $G$ there exists atleast one end vertex in set $\mathrm{K}$.

\subsection{Corollary}

Let $\mathrm{G}$ be a tree with dominating number $\mathrm{c}=\mathrm{c}(\mathrm{G})$, where $2 \leq c \leq\left\lfloor\frac{n}{2}\right\rfloor$. Then $N_{K}(G) \geq N_{K}\left(A^{n, c}\right)$, equality holds if and only if $G \cong A^{n, c}$.

\section{Proof.}

Let $\mathrm{G}$ be $\mathrm{n}$ vertex graph with matching number $\mathrm{m}$. The covering is always contained in dominating set of G. So by lemma $2.1 \mathrm{~m} \geq c$. If $\mathrm{m}=\mathrm{c}$ then by theorem 4.2 we see $N_{K}(G) \geq N_{K}\left(A^{n, c}\right)$., where equality holds if $G \cong A^{n, c}$. If $m>c$ then by theorem 4.2 and theorem 3.1 result follows as,

$$
N_{K}(G)>N_{K}\left(A^{n, m}\right)>N_{K}\left(A^{n, m-1}\right)>\cdots>N_{K}\left(A^{n, c}\right)
$$

\section{$5 N_{K}$-INDEX OF TREES WITH MAXIMUM DEGREE}

Let $\mathrm{t}$ be a positive integer $t \geq 1$. Let $T_{t ; i, j}$ be the tree form a path $P_{2}=x y$ by attaching $t$ distinct pendant vertices, a unique pendant path on $\mathrm{i}$ vertices to $\mathrm{x}$ and connecting $\mathrm{r}$ distinct pendant vertices and unique pendant path on $\mathrm{j}$ vertices to vertex y respectively. Then by corollary 3.2 we see that 


$$
N_{k}\left(G_{t ; i, j}\right)<N_{k}\left(G_{t ; i+1, j-1}\right)
$$

$A_{n, \Delta}$ be a tree obtained from two disjoint stars of same degree $S_{\Delta}$ by joining an edge between their centers and to any pendant vertex of star attaching pendant path of $n-2 \Delta$ vertices, where $n \geq 2 \Delta+1 \geq 7$ For example see Figure 4 .

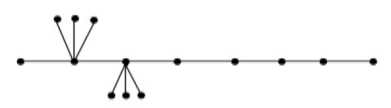

Figure 4: Tree With Maximum Degree

\subsection{Theorem}

Let $\mathrm{G}$ be a tree whose two adjacent vertices have maximum degree $\Delta$, where $n \geq 2 \Delta+1 \geq 7$ Then $N_{k}(G)<$ $N_{k}\left(A_{n, \Delta}\right)$., ith equality if $G \cong A_{n, \Delta}$.

Proof.

Let $\mathrm{G}$ has a maximal $N_{k}$-index such that vertex $\mathrm{x}$ and vertex y are adjacent vertices and share maximum degree $\Delta$. By Theorem 3.1 we have $G \cong G_{t ; i, j}$, where $t=\Delta-2, i \geq$ $j \geq 1$ and $i+j=n-2 \Delta+2$. If $j \geq 2$ then by above argument about $G_{t ; i, j}$ as discussed we have a contradiction.

$$
\begin{gathered}
N_{k}(G) \cong N_{k}\left(G_{t ; i, j}\right)<N_{k}\left(G_{t ; i+1, j-1}\right) \\
\text { Thus } j=1 \text { and } i=n-2 \Delta+1 \text { i.e } G \cong A_{n, \Delta} .
\end{gathered}
$$

\section{REFERENCES}

1. J. A. Bondy, U. S. R. Murty, Graph Theory With Applications, American Elsevier, New York, 1976.

2. A. T. Balaban, Topological Indices Based on Topological Distance in Moleculer Graphs, pure appl. chem., Vol.55, no.2, pp.199-206, 1983.

3. K. Xu, M. Liub, K. C. Dasd, I. Gutmane and B. Furtul, A Survey on Graphs Extremal With Respect to Distance-Based Topological Indices, MATCH Commun. Math. Comput. Chem. Vol.71, pp.461-508, 2014.

4. H. Wiener, Structural Determination of the Paraffin Boiling Points, J. Am. Chem. Soc., Vol.69, pp.17-20, 1947.

5. M. Randic', Novel Molecular Descriptor for Structure-Property Studies, Chem. Phys. Lett. Vol. 211, pp.478-483, 1993.

6. Z. Mihulic and N. Trinajstic, A Graph Theoretical Approach to Strcture-Property Relationship, J. Chem. Educ., vol. 69, pp. 701-712, 1992.

7. O. Ivanciuc, T. S. Balaban and A. T. Balaban, Reciprocal Distance Matrix, Related Local Vertex Invariants and Topological Indices, J. Math. Chem, Vol. 12, pp.309-318, 1993.

8. D. Plavsic, S. Nikolic, N. Trinajstic and Z. Mihalic, On the Harary Index for the Characterization of Chemical Graphs, J. Math. Chem, Vol. 12, pp. 235250, 1993.

9. H. P. Schultz, Topological Organic Chemistry 1, Graph Theory and Topological Indices of Alkanes, J. Chem. Inf. Comput. Sci., vol. 29, pp.227-228, 1989.

10. A. D. Andrey and A. K. Amide, Degree Distance of a Graph: A Degree Analogue of the Wiener Index, J. Chem. Inf. Comput. Sci., vol.34, pp.1082-1086, 1994.

11. A. T. Balaban, Topological Indices Based on Topological Distance in Moleculer Graphs, pure appl. chem., Vol. 55, no.2, pp.199-206, 1983.

12. A. Ilic', G. Yu and L. Feng, On the Eccentric Distance Sum of Graphs, J. Math. Anal. Appl., vol.381, pp.590600, 2011.

13. H. Qu and S. Cao, On the Adjacent Eccentric Distance Sum Index of Graphs, PLoS ONE Academic Editor: Vince Grolmusz, Mathematical Institute, HUNGARY, vol.10, no. 6, pp.1-12, 2015.

14. A. M. Naji and N. D. Soner, The k-Distance Degree Index of a Graph, Palestine Journal of Mathematics, vol. 7, no.2, pp.676-687, 2018.

15. A. M. Naji and N. D. Soner, The k-Distance Degree Index of Corona, Neighborhood Corona Products and Join of Graphs, International J.Math. Combin, Vol. 4, pp.91-102, 2017.

16. I. Gutman, B. Furtula, V. Markovic', B. Gliš̆c', Z. Naturforsch, Alkanes With Greatest Estrade Index, Vol.62a, pp.495, 2007.

17. M. Fischermann, A. Hoffmann, D. Rautenbach, L. Sze'kely, L. Volkmann, Wiener Index Versus Maximum Degree In Tree, Discrete Appl. Math,vol.122, pp.127-137, 2002.

18. S. Simic', D. Tosĭc', The Index Of Tree With Specified Maximum Degree, MATCH Commun. Math. Comput. Chem, vol.54, pp.351-362, 2005.

19. D. Stevanovic' and A. Ilic', Distance Spectral Radius of Trees With Fixed Maximum Degree, Electronic Journal of Linear Algebra, vol.20, 2010. 